\title{
Care Pathway of RPE65-Related Inherited Retinal Disorders from Early Symptoms to Genetic Counseling: A Multicenter Narrative Medicine Project in Italy
}

\author{
Francesca Simonelli ${ }^{1}$ \\ Andrea Sodi ${ }^{2}$ \\ Benedetto Falsini ${ }^{3,4}$ \\ Giacomo Bacci ${ }^{5}{ }^{5}$ \\ Giancarlo larossi ${ }^{6}$ \\ Valentina Di lorio' \\ Dario Giorgio ${ }^{2}$ \\ Giorgio Placidi ${ }^{3,4}$ \\ Assia Andrao ${ }^{7}$ \\ Luigi Reale iD ${ }^{8}$ \\ Alessandra Fiorencis ${ }^{8}$ \\ Manar Aoun ${ }^{9}$ \\ 'Eye Clinic, Multidisciplinary Department of \\ Medical, Surgical and Dental Sciences, \\ University of Campania "L. Vanvitelli", \\ Naples, Italy; ${ }^{2}$ Department of Neuroscience, \\ Psychology, Drug Research and Child \\ Health, University of Florence, Florence, \\ Italy; ${ }^{3} \mathrm{UOC}$ Oftalmologia, Fondazione \\ Policlinico Universitario A. Gemelli IRCCS, \\ Rome, Italy; ${ }^{4}$ Dipartimento Testa-collo \\ e organi di senso, Università Cattolica del \\ Sacro Cuore, Rome, Italy; ${ }^{5}$ Paediatric \\ Ophthalmology Unit, Children's Hospital "A. \\ Meyer", University of Florence, Florence, \\ Italy; ${ }^{6}$ Ophthalmology Department, \\ Bambino Gesù IRCCS Paediatric Hospital, \\ Rome, Italy; ${ }^{7}$ Retina Italia Onlus Association, \\ Milan, Italy; ${ }^{8}$ Healthcare Department, \\ Fondazione ISTUD, Milan, Italy; ${ }^{9}$ Medical \\ Department, Novartis Farma, Origgio, Italy
}

Correspondence: Luigi Reale Healthcare Department, Fondazione ISTUD, via Paolo Lomazzo 19, Milano, 20124, Italy

Tel +390323933801

Email Ireale@istud.it

Francesca Simonelli Tel +3908I770450 I

Email francesca.simonelli@unicampania.it
Purpose: Timely detection and multidisciplinary management of RPE65-related inherited retinal disorders (IRDs) can significantly improve both disease management and patient care. Thus, this Narrative Medicine (NM) project aimed to investigate the evolution of the care pathway and the expectations on genetic counseling and gene therapy by patients, caregivers, and healthcare professionals.

Patients and Methods: This project was conducted between July and December 2020, involving five Italian eye clinics specialized in IRDs, targeted pediatric and adult patients, their caregivers, attending retinologists and multidisciplinary healthcare professionals. Narratives and parallel charts, together with a sociodemographic survey, were collected through the project webpage. In-depth interviews were conducted with Patient Association (PA) members and multidisciplinary healthcare professionals. All data were entered into the Nvivo Software for coding and analysis.

Results: Three pediatric and five adult patients with early-onset RPE65-related IRDs as well as eight caregivers were enrolled; 11 retinologists globally wrote 27 parallel charts; in-depth interviews were done with five multidisciplinary healthcare professionals and one PA member. Early diagnosis remains challenging, and patients reported to have changed up to 10 healthcare professionals before accessing their specialized center. Despite the oftentimes lack of awareness of patients and caregivers on the purpose of genetic testing, participants generally consider gene therapy as a therapeutic chance and a historic breakthrough for the management of RPE65-related IRDs. Well-organized networks to support the patient's referral to specialized centers - as well as a proper communication of the clinical and genetic diagnosis and the multidisciplinary approach - emerge as crucial aspects in facilitating an early diagnosis and management and a timely initiation of the rehabilitation pathway.

Conclusion: The project investigated the RPE65-related IRDs care pathway while integrating the different perspectives involved through NM. The analysis explored the patient's pathway in Italy and confirmed the need for a well-organized network and multidisciplinary care while highlighting several preliminary areas of improvement in the management of RPE65-related IRDs.

Keywords: IRDs management, multidisciplinary, inherited retinal dystrophies, diagnosis, patient's pathway

\section{Introduction}

Inherited retinal dystrophies (IRDs) are a group of genetically and clinically heterogeneous degenerative conditions ${ }^{1}$ affecting approximately 1 in 2-3000 people 
worldwide. $^{2}$ IRDs related to mutations involving the RPE65 gene are characterized by a progressive loss of photoreceptor cells and visual function impairment, resulting in an irreversible visual decline ${ }^{3}$ and potential blindness; ${ }^{4}$ retinitis pigmentosa $(\mathrm{RP})^{5}$ and Leber congenital amaurosis (LCA) ${ }^{6}$ are the most common phenotypes.

The deterioration of visual acuity and peripheral vision follows early symptoms: visual impairment in different luminance conditions, night blindness, and nystagmus. ${ }^{7,8}$ While IRDs management remains mainly support-oriented with a focus on monitoring, education, and counseling, ${ }^{3}$ gene therapy represents a novel potential treatment ${ }^{3,9-11}$ as no treatments yet could slow or stop the progression of sight loss. ${ }^{12}$ Since the age of onset ranges from early childhood to middle age, a timely detection of IRDs ${ }^{13,14}$ and an optimal multidisciplinary management ${ }^{15}$ can significantly influence the improvement of the patient's quality of life and offer therapeutic perspectives: an early diagnosis is critical to proper visual rehabilitation, as well as for genetic diagnosis and counseling and gene replacement therapies. ${ }^{16,17}$ In particular, referral to highly specialized centers ensures multidisciplinary and personalized care, ${ }^{18}$ while a genetic diagnosis is critical to establish eligibility for an optimal gene therapy. ${ }^{17}$

Given the lack of data related to care pathways and patient's quality of life, ${ }^{19}$ patient self-reporting has become crucial for the development of a suitable multidisciplinary management strategy. ${ }^{20}$ Moreover, as affirmed in other studies, ${ }^{21}$ it is crucial to investigate methodologies that can gather patient experience that mere analysis of clinical pathway could not determine.

The World Health Organization (WHO) highlighted the significance of researching the illness experience more broadly $^{22}$ to inform clinical practice, ${ }^{23}$ and recommended the use of narrative research.

As described in other studies, ${ }^{24,25}$ Narrative Medicine (NM) deals with illness narratives ${ }^{26}$ to pursue the integration of the biomedical sphere with the individual and social experience of a condition. ${ }^{27}$ Within the research, NM helps identify feasible interventions and implement the care pathway, by integrating the perspectives of all the factors involved; ${ }^{28,29}$ its findings have been increasingly used to improve the quality of care, ${ }^{30,31}$ also in the clinical genetics practice. ${ }^{32}$

The NM project "BIRDS - The Beat of IRD Stories" aimed to study the RPE65-related IRDs illness experience through the analysis of narratives (a) to investigate the evolution of the care pathway and the expectations about genetic counseling and gene therapy as experienced by patients, caregivers, and healthcare professional; (b) to reveal the impact of these conditions on the personal and social quality of life; and finally (c) to provide insights for a better knowledge and clinical practice on RPE65-related IRDs. This study focuses on the first purpose of the project; to the best of our knowledge, no other study investigated the RPE65-related IRDs care pathway while considering at the same time the perspectives of underage and adult patients, caregivers, retina specialists and multidisciplinary healthcare professionals.

\section{Methods}

\section{Research Design and Setting}

The project was conducted between July and December 2020, targeting pediatric and adult patients with an RPE65-related IRD, caregivers, retina specialists, and other healthcare professionals from multidisciplinary teams involved in providing care. Five Italian eye clinics specialized in IRDs - ie, two pediatric hospitals and three university hospitals (Supplementary file 1) - were involved in the enrollment of participants. In July 2020, researchers from "Istituto Studi Direzionali" (ISTUD) Foundation trained the Steering Committee - which involved one Patient Association (PA) member and five retinologists specialized in IRDs - on NM and on the project's purposes, design, and investigation tools; afterward, researchers invited them to engage patients and caregivers in participating in the research by accessing the project webpage [http://www.medicinanar rativa.eu/birds].

A clinical RPE65-related IRD diagnosis, determined at the reference medical center, or the caregiving of a person with this condition were the eligibility criteria for pediatric and adult patients and caregivers, together with the willingness to share their experience on the care pathway; thus, the ability to communicate in Italian was also critical.

\section{Data Collection}

Researchers ensured the survey accessibility by following the Web Content Accessibility Guidelines (WCAG) 2.1; ${ }^{33}$ furthermore, patients could share their experience either by writing or recording an audio file, to address their low vision challenges and obtain age-appropriated support in writing, if underage. Narratives were anonymously collected through the Alchemer platform, available on the project webpage; subsequently, raw narratives were downloaded as Microsoft Excel spreadsheets. An illness plot ${ }^{34}$ ie, a plot characterized by evocative words to encourage 
individual expression ${ }^{35}$ and aimed to retrace the illness experience in order to identify its evolution over time was formed based on the written narrative for patients and caregivers, together with a sociodemographic survey.

Researchers gathered retina specialists' care experience through the parallel chart $^{36}$ - ie, a personal notebook where the retinologists could record their thoughts and feelings in plain language, in addition to the technical reports. ${ }^{37}$ The patients described in parallel charts could not be the same ones participating in the projects, as well as the patients' caregivers could not be the same ones sharing their experience.

These investigation tools (Supplementary file 2) were designed for the three groups of participants to address common aspects: (a) the onset of symptoms, the pathway toward the clinical diagnosis, and the clinical encounter; (b) the experience of genetic counseling and the participants' expectations about gene therapy; and (c) the evolution of the care relationship over time and the target areas to be improved.

Furthermore, researchers performed an in-depth interview $^{38}$ with five multidisciplinary healthcare professionals involved in IRD management and one PA member, to further help care pathway-related issues to emerge; each participant approved the final transcript before the analysis.

The investigation tools, summarized in Table 1, were designed by two ISTUD researchers with different academic backgrounds and were reviewed within the Steering Committee to reduce any cognitive bias.

\section{Ethical Considerations}

The project was carried out according to the Declaration of Helsinki and approved by the Ethical Committee of the "Luigi Vanvitelli" University Hospital (Naples, Italy) in September 2020. The retinologists involved obtained a written informed consent to participate from the parents of underage participants during the first project meeting; adult participants provided their web-based informed consent after briefing on the research purposes and confidential data processing procedures according to the General Data Protection Regulation of the European Union 2016/ $679^{39}$ and the Italian Law 196/2003. ${ }^{40}$ All informed consents included the publication of anonymized responses and narratives (Supplementary file 3).

\section{Analysis}

Survey data was analyzed through descriptive statistics; answering questions or filling in fields in the illness plots and the parallel charts was not mandatory, thus the varying sample sizes. Researchers entered anonymous narratives into the Nvivo software for coding and analysis; ${ }^{41}$ three narratives for each group and one in-depth interview were collectively coded to assess the consistency across team members and then reviewed during weekly peer debriefings, to limit any cognitive bias. Researchers employed open interpretive coding to identify and analyze the emerging topics in the narratives and in-depth interviews.

The Steering Committee examined the results to collectively address any issues emerged and the data interpretation. Researchers followed the Consolidated Criteria for Reporting Qualitative Research (COREQ) reporting guidelines. ${ }^{42}$

\section{Results}

Eight patients with an early RPE65-related IRD onset participated in the project in writing, together with eight caregivers (parental or partner); 11 retinologists specialized in IRDs globally wrote 27 parallel charts. One PA member and five multidisciplinary healthcare professionals - ie, two

Table I Investigation Tools Used in the Project

\begin{tabular}{|l|l|}
\hline Category of Participants & Investigation Tools \\
\hline Underage and adult patients & $\begin{array}{l}\text { Sociodemographic survey } \\
\text { Illness plot, to collect written narratives } \\
\text { Audio track, to present and promote audio narratives }\end{array}$ \\
\hline Caregivers & $\begin{array}{l}\text { Sociodemographic survey } \\
\text { Illness plot, to collect written narratives }\end{array}$ \\
\hline Retinologists & $\begin{array}{l}\text { Sociodemographic survey } \\
\text { Parallel chart }\end{array}$ \\
\hline Multidisciplinary healthcare professionals and Patient Association member & $\begin{array}{l}\text { Sociodemographic survey } \\
\text { In-depth interview }\end{array}$ \\
\hline
\end{tabular}


psychologists, two genetic counselors, and one orientation and mobility instructor - participated in the in-depth interviews.

Tables 2 and 3 summarize the sociodemographic data of participants; non-responders are included as a separate category.

Figure 1 provides a flowchart to reconstruct the RPE65-related IRDs care pathway from the patients' and caregivers' narratives.

Quantitative data are presented as median (range). The following four subsections illustrate the main findings: (a) the onset of the condition, the pathway toward the clinical diagnosis, and the first clinical encounter in a IRDs specialized center; (b) the genetic counseling experience and the personal expectations about gene therapy; (c) the evolution of the care relationship over time and the target areas to be improved; and (d) insights and proposals for the improvement of the care pathway from the in-depth interviews. Figures 2-5 and Tables 4 and 5 report quotes from narratives, parallel charts, and in-depth interviews. Supplementary file 3 provides four narratives from participants (in English). Researchers reduced the reidentification risk by applying different codes from those used to identify participants during data collection.

\section{From the Onset of Symptoms to the Beginning of the Care Pathway}

Patients showed the first signs of visual impairment at 2 years and 3 months of age (0,5-6 years). Light gazing and the

Table 2 Sociodemographic Data of Patients, Caregivers, and Patients Described in Parallel Charts

\begin{tabular}{|c|c|c|c|}
\hline & Patients $(\mathrm{N}=\mathbf{8})$ & Caregivers $(\mathrm{N}=8)$ & $\begin{array}{l}\text { Patients in Parallel Charts } \\
\qquad(\mathbf{N}=\mathbf{2 7})\end{array}$ \\
\hline \multicolumn{4}{|l|}{ Gender } \\
\hline Female & $6(75 \%)$ & $6(75 \%)$ & 12 (44\%) \\
\hline Male & $2(25 \%)$ & $2(25 \%)$ & $15(56 \%)$ \\
\hline \multicolumn{4}{|l|}{ Age (yrs) } \\
\hline Median (range) & $26(8-63)$ & $44(31-70)$ & $17(5-65)$ \\
\hline$<18$ & $3(38 \%)$ & - & $15(56 \%)$ \\
\hline$>18$ & $5(62 \%)$ & $8(100 \%)$ & $12(44 \%)$ \\
\hline \multicolumn{4}{|l|}{ Geographic residence } \\
\hline Northern Italy & $3(38 \%)$ & $2(24 \%)$ & - \\
\hline Central Italy & $4(50 \%)$ & $4(50 \%)$ & - \\
\hline Southern Italy & I (I2\%) & I (I3\%) & - \\
\hline Non-responders & - & I (13\%) & - \\
\hline \multicolumn{4}{|l|}{ Education } \\
\hline Elementary school & I (I2\%) & - & $7(26 \%)$ \\
\hline Middle school & - & I (I2\%) & $4(15 \%)$ \\
\hline High school & I (I2\%) & $3(38 \%)$ & $4(15 \%)$ \\
\hline Bachelor/Master & $3(38 \%)$ & $3(38 \%)$ & $3(1 \mid \%)$ \\
\hline Non-responders & $3(38 \%)$ & I (I2\%) & $9(33 \%)$ \\
\hline \multicolumn{4}{|l|}{ Employment status } \\
\hline Student & $4(50 \%)$ & - & 16 (59\%) \\
\hline Working & $3(38 \%)$ & $6(76 \%)$ & $10(37 \%)$ \\
\hline Not working & - & - & - \\
\hline Retired & - & I (I2\%) & I (4\%) \\
\hline Non-responders & I (I2\%) & I (I2\%) & - \\
\hline \multicolumn{4}{|l|}{ Marital status } \\
\hline Single & $6(75 \%)$ & I (I2\%) & 18 (67\%) \\
\hline Married & $2(25 \%)$ & $5(64 \%)$ & 7 (26\%) \\
\hline Separated & - & I (I2\%) & $2(7 \%)$ \\
\hline Non-responders & - & I (I2\%) & - \\
\hline
\end{tabular}

Notes: Data are presented as $\mathrm{n}(\%)$ or median (range). 
Table 3 Sociodemographic Data of the Retinologists and Multidisciplinary Healthcare Professionals Interviewed

\begin{tabular}{|c|c|c|}
\hline & $\begin{array}{l}\text { Retinologists } \\
(\mathrm{N}=11)\end{array}$ & $\begin{array}{c}\text { Multidisciplinary } \\
\text { Healthcare } \\
\text { Professionals }(\mathrm{N}=6)\end{array}$ \\
\hline \multicolumn{3}{|l|}{ Gender } \\
\hline Female & $5(45 \%)$ & $5(83 \%)$ \\
\hline Male & $6(55 \%)$ & I (17\%) \\
\hline \multicolumn{3}{|l|}{ Age (yrs) } \\
\hline Median (range) & $42(32-64)$ & $54(49-67)$ \\
\hline \multicolumn{3}{|l|}{ Geographic residence } \\
\hline Northern Italy & - & $2(33 \%)$ \\
\hline Central Italy & $8(73 \%)$ & $4(67 \%)$ \\
\hline Southern Italy & $3(27 \%)$ & - \\
\hline Non-responders & - & - \\
\hline \multicolumn{3}{|l|}{ Professional activity duration (yrs) } \\
\hline Median (range) & $16(6-41)$ & $23(19-35)$ \\
\hline \multicolumn{3}{|l|}{ Specialization } \\
\hline Ophthalmologist & $8(73 \%)$ & I (17\%) \\
\hline Pediatric Ophthalmologist & I (9\%) & \\
\hline Orthoptist & $2(18 \%)$ & \\
\hline Medical Geneticist & & I (17\%) \\
\hline Rehabilitation technician & & I (17\%) \\
\hline Psychologist & & $2(32 \%)$ \\
\hline Patient Association member & & $\mathrm{I}(17 \%)$ \\
\hline \multicolumn{3}{|l|}{ Workplace } \\
\hline Hospital & $2(18 \%)$ & \\
\hline University Hospital & $9(82 \%)$ & $2(33 \%)$ \\
\hline Other & & $4(67 \%)$ \\
\hline
\end{tabular}

Notes: Data are presented as $\mathrm{n}(\%)$ or median (range).

inability to see objects in luminance change conditions were reported as the critical signs identified during early childhood in $57 \%$ of patients' narratives (Figure 2), followed by balance and coordination issues and night blindness (both 43\%). Parental caregivers reported light gazing and lack of eye contact, both present in $83 \%$ of the narratives, and night blindness $(50 \%)$ as the main signs of visual impairment.

Pediatricians and ophthalmologists were the first professionals to be consulted in $57 \%$ and $43 \%$ of patients' narratives, respectively. Patients reported to have changed up to 5 (median, 2.5; range, 1-5) medical centers and up to 10 (median, 2; range $0-10$ ) healthcare professionals before accessing the IRDs specialized center; in $75 \%$ of patients' narratives, interregional mobility emerged as highly important in order to access the specialized center in terms of IRDs knowledge and diagnostic capabilities for pediatric patients

My parents started travelling around Italy looking for doctors who could explain what was going on. Pediatricians said that my mother was too anxious [...]. The diagnosis came only after many doctors and many trips, Patient 006.

Fifty percent of patients reported communication issues upon the clinical diagnosis, mainly highlighting disorientation

They told my mother that I needed psychological support to cope with the condition, and that the doctor could help me. I cried. I just cried. [...] I cannot say who made more mistakes: the doctors I met, so little empathetic, or the school and its bureaucratic procedures, or my family, which raised and treated me as "normal". Some categories should be included with caution in a child's life, but it must be done indeed, to avoid worse disorientation, Patient 001.

Or a lack of therapeutic proposals

The trips ended when the lapidary diagnosis arrived: "Retinitis pigmentosa. There is no cure", Patient 006.

Within the parallel charts, retinologists indicated the need for a therapeutic perspective (28\%), the concern for the evolution of the condition ( $40 \%$ ), and the trust in the IRDs specialized center $(32 \%)$ as the main issues expressed by patients and caregivers during the first clinical encounter (Table 4). In addition, they described the communication strategies employed to start an adequate care pathway: active listening and empathy emerged as crucial in $50 \%$ of the parallel charts, the effort to provide a proper and correct information in $42 \%$ and the self-identification with patients or parental caregivers in $18 \%$.

\section{Genetic Counseling and Expectations About Gene Therapy}

All patients reported to have undergone genetic counseling. Nonetheless, $57 \%$ accessed the specialized center without knowing the importance of genetics, and without having undergone a genetic test after the clinical diagnosis (Figure 3), while $43 \%$ referred to be too young to understand its purpose entirely. Conversely, $25 \%$ of caregivers reported having become aware of the aim of genetic test only recently, and $75 \%$ considered it a chance to receive definite answers and a complete diagnosis.

Retinologists referred to have proposed the genetic test only in $56 \%$ of the parallel charts, highlighting its importance for the prognosis and the therapeutic pathway

An anchor. The genetic test can provide more information on the condition, and potentially on the prognosis and the chances of care, Parallel chart 010. 


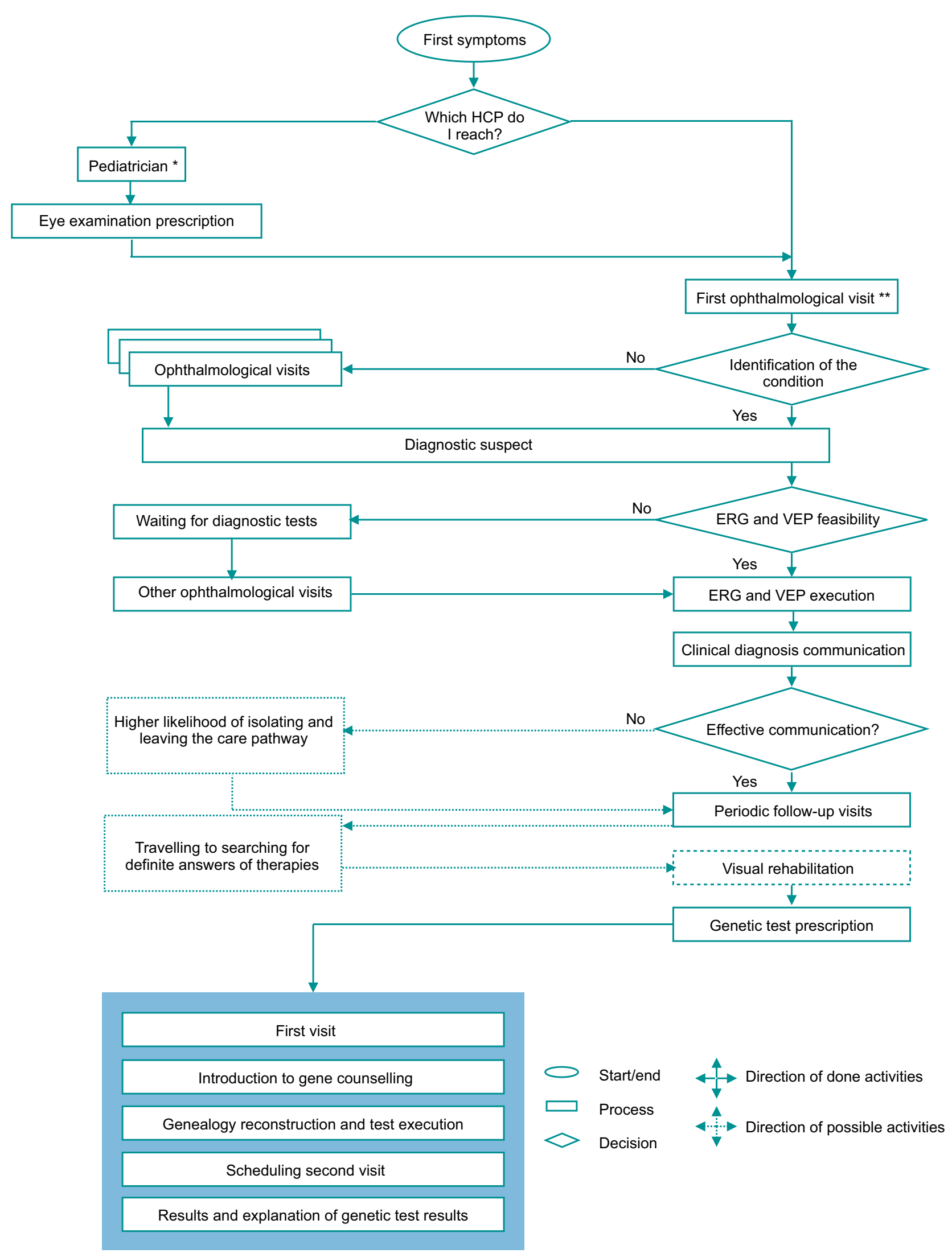

Figure I Care pathway flowchart as deduced from patient and caregiver narratives. *Firstly addressed professionals in $57 \%$ of patient narratives. **Firstly addressed professionals in $43 \%$ of patient narratives.

Abbreviations: HCP, Healthcare professional; ERG, Electroretinogram; VEP, Visually evoked potentials. 


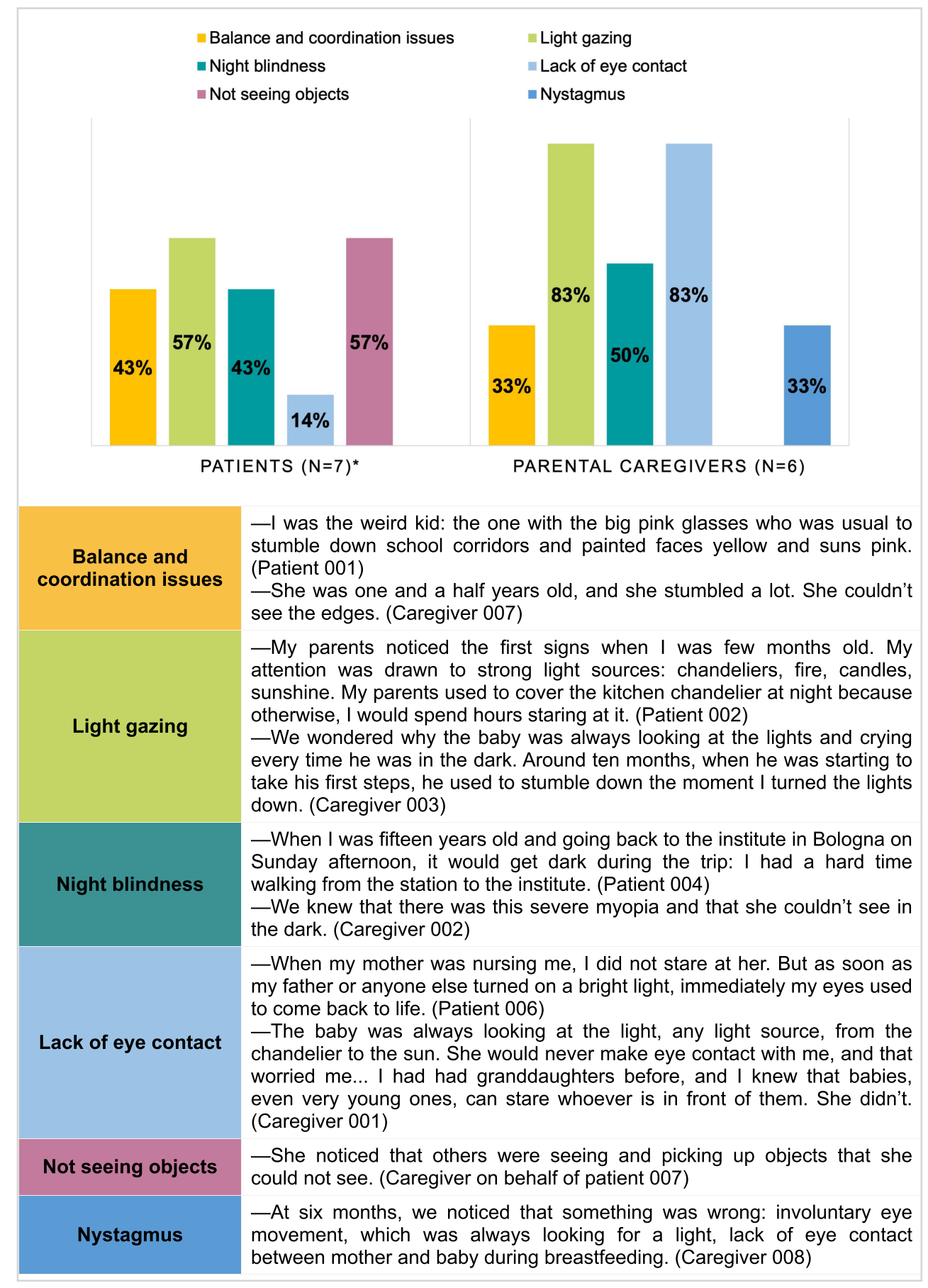

Figure 2 First RPE65-related IRDs signals reported by patients and parental caregivers: distribution and quotes from narratives. $*$ Non-responses $=1$.

Gene therapy represented the hope for preventing the progression of sight loss in $83 \%$ of patients' and in $75 \%$ of caregivers' narratives (Figure 4), and a chance for visual improvement in $17 \%$ of patients' and $25 \%$ of caregivers' narratives.

All retinologists considered gene therapy a historic breakthrough for the therapeutic management of RPE65related IRDs
A turning point for previously incurable conditions, Parallel chart 026.

Thus, in $67 \%$ of the parallel charts, retinologists defined the access to gene therapy as still limited to a few patients and requiring to be further clarified and implemented 


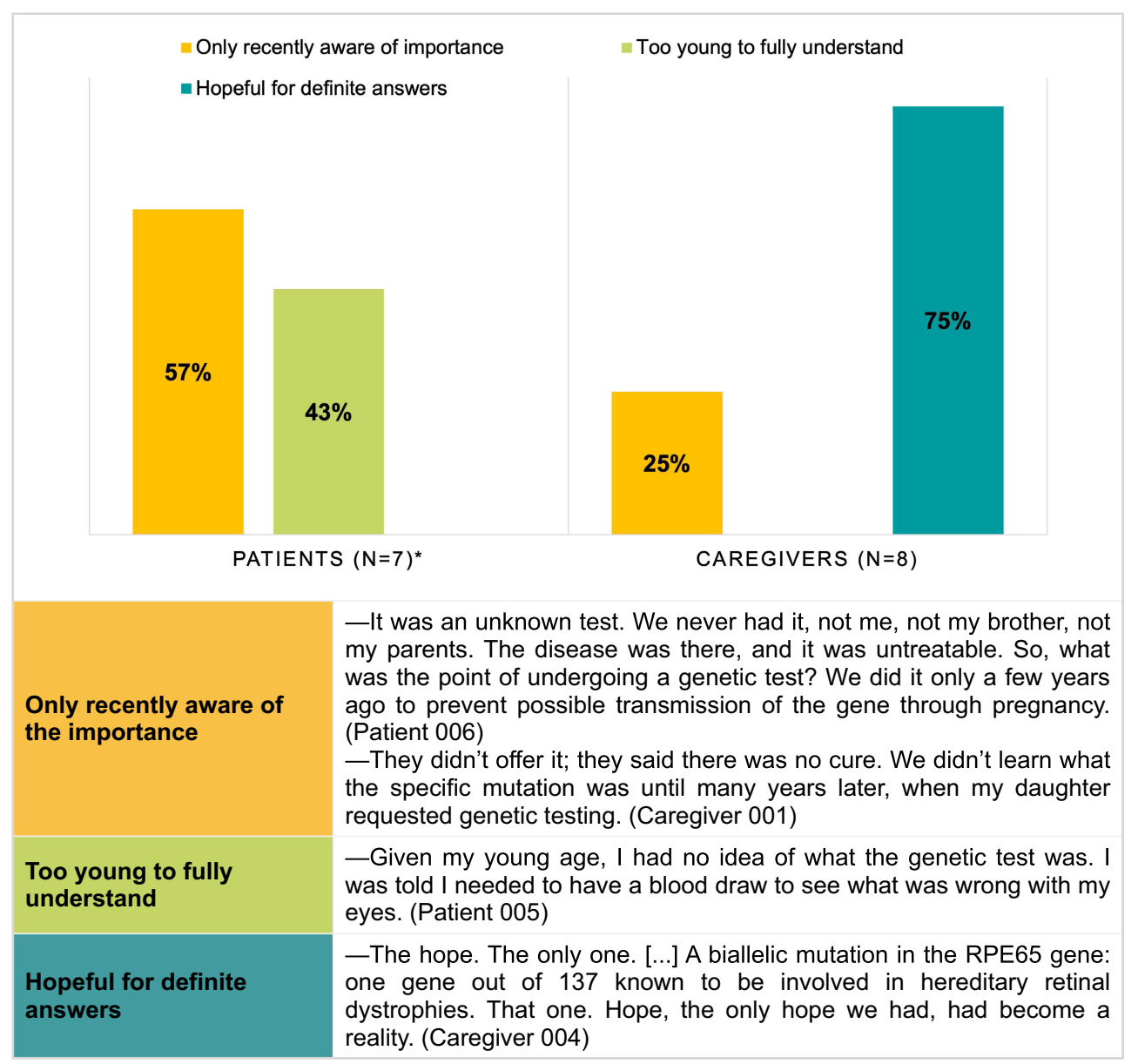

Figure 3 Genetic test in patients' and caregivers' perspectives: distribution and quotes from narratives. *Non-responses $=1$.

Gene therapy represents the future of medicine in several areas; access to therapies should be extended to all. However, this is currently a utopian thought. We need more research to make it real, Parallel chart 005.

Also at a communication and professional training level

We have a lot to do in terms of organization, communication and training, Parallel chart 014.

\section{Evolution of Care Relationship and Target Areas to Be Improved}

Thirty-three percent of patients stated to hope to access gene therapy; conversely, $67 \%$ reported a feeling of selfdelusion

I go straight my way with the strategies I have today, and I rarely indulge in fantasies. If I perceive therapy as a fantasy, it remains in its place ... But if it were to become a desire, everything would crash down. I cannot afford it, Patient 001.
However, 63\% reported to trust the professionals of their specialized center, and $75 \%$ expressed a positive evolution of the care relationship

It gives me much confidence. It makes me feel considered - I have never experienced that before, Patient 002.

Retinologists confirmed the positive evolution of the care relationship in $67 \%$ of the parallel charts

We monitored the rehabilitation path. The child appeared more confident in his visual potential and increased his self-esteem, Parallel chart 004.

Moreover, they reported the hope for their patients to access gene therapy (19\%) and, conversely, their increasing issues in performing everyday tasks due to visual impairment (15\%). The provision of an effective support $(46 \%)$, the improvement of the patients' autonomy and quality of life $(42 \%)$ and the promotion of their awareness $(12 \%)$ emerged as the retinologists' main goals of care. 


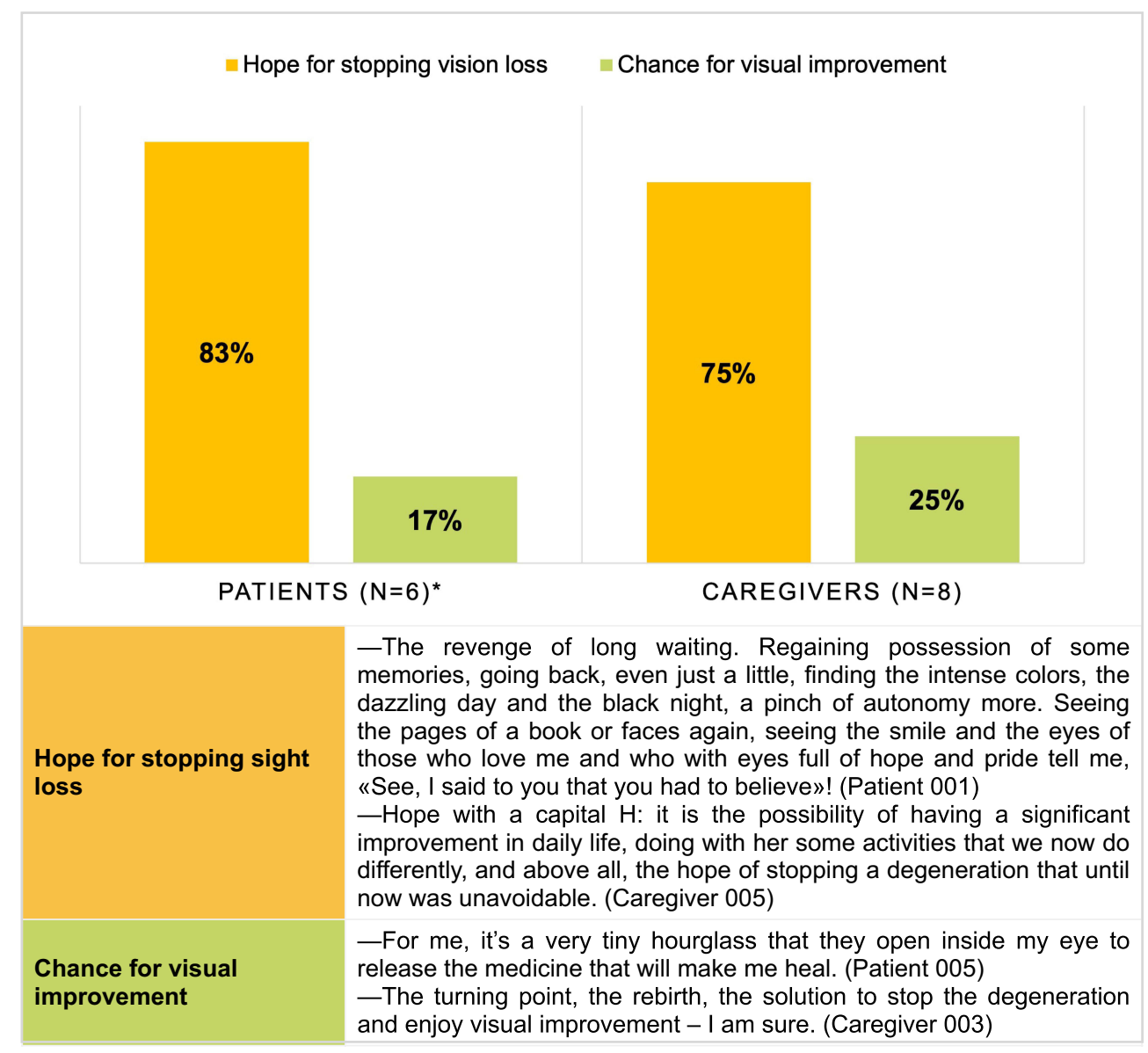

Figure 4 Patients and caregivers' expectations over gene therapy: distribution and quotes from narratives. $*$ Non-responses $=2$.

With respect to different care pathway experiences (Figure 5), patient preferences were as follows: more involvement $(50 \%)$ and more support on the part of healthcare professionals $(63 \%)$, an earlier genetic testing $(38 \%)$, and an earlier discovery of the gene therapy (38\%). Similarly, preferences of caregivers were more support (38\%), the possibility to share their caregiving experience with other families experiencing the same situation (13\%), earlier genetic testing (50\%), and an earlier discovery of the gene therapy $(25 \%)$.

The survey revealed that patients find information on their condition mainly at the centers specialized in IRDs (71\%), while believing that a greater awareness of IRDs clinical manifestations among the general public is highly needed (86\%). Furthermore, patients stated that a PA should provide updated and detailed information on the management of IRDs and the therapeutic progress (83\%) and suggestions aimed at improving quality of life and autonomy (50\%). Patients also suggested that PA members should encourage or organize patients' self-help groups
$(50 \%)$. Of the caregivers, $83 \%$ reported that a PA should provide valuable contacts and information sources about the condition, $33 \%$ sought recommendations regarding the lifestyle, and 33\% thought that they should connect patient both among themselves and with the specialized centers. According to clinicians, educational programs on IRDs should be highly recommended for patients (64\%), as well as for ophthalmologists (55\%) and pediatricians $(45 \%)$.

\section{Insights from in-Depth Interviews}

Three main topics transversely emerged from the in-depth interviews done with the PA member and the multidisciplinary healthcare professionals (Table 5):

(a) All interviewees emphasized that the information provided to patients and caregivers from the diagnostic suspect onward should be correct and that psychological support should be guaranteed upon the diagnosis and throughout the care pathway. 


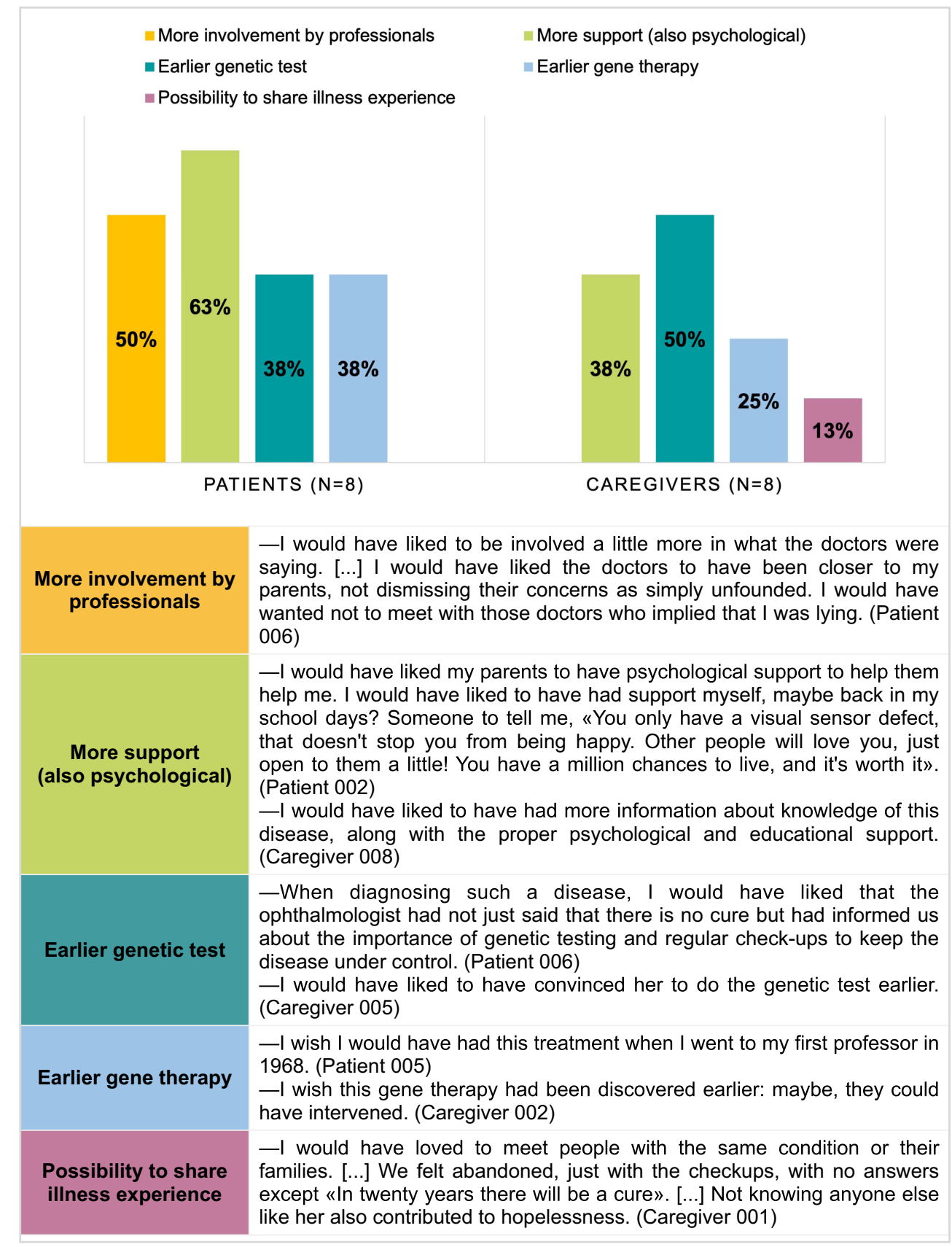

Figure 5 What patients and caregivers would have wanted thinking the care pathway retrospectively: distribution and quotes from narratives.

Psychologists suggested that communication should be evaluated on a case-by-case basis. The PA member highlighted that an "abrupt" communication can proactivity impact the patient along her rehabilitation pathway, which is often not timely accessed and properly acknowledged, as outlined by the orientation and mobility instructor.

(b) Geneticists stressed that, on the one hand, patients and caregivers often ignore the role of genetic counseling in exploring therapeutic options and that, on the other, such counseling should be integrated within a multidisciplinary approach. Interviewees reported criticalities in accessing genetic testing: the limited number of centers specialized in IRDs and equipped with new generation technologies for genetic testing and challenging waiting times delay both the rehabilitation and the therapeutic pathway. 
Table 4 The First Diagnostic Visit at a IRDs Specialized Center: Distribution and Quotes from Parallel Charts

\begin{tabular}{|c|c|}
\hline \multicolumn{2}{|c|}{ Main issues expressed by patients and caregivers } \\
\hline Concern $40 \%$ & $\begin{array}{l}\text {-They were very concerned about his autonomy and the possibility of leading a normal life in the future. (Parallel } \\
\text { chart 024) }\end{array}$ \\
\hline Trust $32 \%$ & $\begin{array}{l}\text {-Parents looked at me as a reference person to confide their fears and thoughts on the evolution of the } \\
\text { condition. (Parallel chart } 010 \text { ) }\end{array}$ \\
\hline $\begin{array}{l}\text { Need for a therapeutic } \\
\text { perspective } 28 \%\end{array}$ & -They told me that they confided in me to provide a cure soon. (Parallel chart $0 \mathrm{II}$ ) \\
\hline \multicolumn{2}{|c|}{ Retinologists' communication strategies } \\
\hline Self-identification $18 \%$ & $\begin{array}{l}\text {-I appealed to my role as a physician and as a parent to fully understand the issues of the child and her family. } \\
\text { I tried to integrate the diagnosis by explaining that early-onset retinal dystrophies are the most complex to } \\
\text { investigate, because of their confounding and, often, poorly specific clinical features. (Parallel chart 00I) }\end{array}$ \\
\hline $\begin{array}{l}\text { Active listening and empathy } \\
50 \%\end{array}$ & $\begin{array}{l}\text {-I listened to him with as much empathy as possible, not having the chance to catch his gaze. I tried to make him } \\
\text { feel that I was close to him. (Parallel chart 014) }\end{array}$ \\
\hline Correct information $42 \%$ & $\begin{array}{l}\text {-I tried to convey some concepts that, in my opinion, were critical for her, as well as for all the other patients with } \\
\text { these diseases. I tried to explain that a precise diagnosis is fundamental to receive the proper therapy. And I tried to } \\
\text { explain that this therapy, in her case, would be applicable in the not-too-distant future. (Parallel chart 015) }\end{array}$ \\
\hline
\end{tabular}

Table 5 Main Topics Emerged from the Patient Association Member and the Multidisciplinary Healthcare Professionals: Quotes from the in-Depth Interviews

\begin{tabular}{|c|c|}
\hline $\begin{array}{l}\text { Communication of } \\
\text { IRDs }\end{array}$ & $\begin{array}{l}\text {-Communication should be evaluated on a case-by-case basis. I believe that communication should be done when both } \\
\text { premises and scientific certainties are present; moreover, we should psychologically support the patient. The double } \\
\text { possibility of success and failure should always be considered. It is necessary to work on both because "you can die of } \\
\text { happiness": what human beings fear the most is change, and it has been said that we do not react well to change, even if } \\
\text { positive, because we can still be afraid of it. (Interviewee 003) } \\
\text {-We still have situations in which the communication of the diagnosis is made abruptly. They say, "Unfortunately, there are } \\
\text { no cures for the disease, blindness could arrive, although we don't know when". Verbal "violence" takes away any hope. } \\
\text { [...] The main problem after the diagnosis is the psychological aspect. Suppose the diagnosis is communicated together } \\
\text { with the possibility of recovery. In that case, you deal with it somehow, but if it is offered without any chance of recovery, } \\
\text { people do not even attend control visits anymore; "Why should I even undergo any control?", they ask. [...] This is } \\
\text { a significant shortcoming of our system, the absence of a psychologist upon the communication of the diagnosis. } \\
\text { (Interviewee 004) }\end{array}$ \\
\hline Genetic & $\begin{array}{l}\text {-A genetic investigation untrammeled from a multidisciplinary clinical context is unimportant. It represents highly complex } \\
\text { information that must be managed by competent professionals, for example when reconstructing a family tree; genetic } \\
\text { counseling should give correct and proper information. (Interviewee 005) } \\
\text {-The waiting time for genetic testing should be reduced. Surely, we should invest in research. [...] I am alone, I can hardly } \\
\text { provide answers to everybody. Waiting times are long; we need to increase professional training. (Interviewee 002) }\end{array}$ \\
\hline $\begin{array}{l}\text { Lack of knowledge of } \\
\text { IRDs }\end{array}$ & $\begin{array}{l}\text {-No one knows about retinal dystrophies, because they are rare diseases that you do not encounter often. At first, you } \\
\text { do not understand what's happening to you; these diseases show up in developmental age, we do not always realize that } \\
\text { something is wrong. The moment we realize something is wrong, formulating the diagnosis is difficult. The general } \\
\text { practitioner is not always able to refer the patient to the specialists who should examine her. Unfortunately, the } \\
\text { ophthalmologist is not always able to understand immediately what the problem is. These are diseases that show up early, } \\
\text { and they are not always recognized in time. (Interviewee 004) }\end{array}$ \\
\hline
\end{tabular}

(c) All interviewees highlighted the importance of a continuous work on the awareness of IRDs among both the public opinion and healthcare professionals non-specialized in IRDs, as well as other professionals potentially involved in multidisciplinary care teams - also given the fact that 
the onset of these conditions occurs during the developmental age.

Furthermore, the interviewees suggested that an effective care pathway should require (a) a well-organized network between centers specialized in IRDs and on a national level and more clinical geneticists specialized in this field; (b) shorter waiting times for genetic testing and rehabilitation; and (c) a multidisciplinary care approach with more training on IRDs for professionals and more room for psychological support.

\section{Discussion}

This project represents the first effort to investigate RPE65-related IRDs care pathway in Italy through NM that simultaneously addresses the perspectives of patients, caregivers, and attending physicians and collecting insights from the genetic counselors, psychologists, and orientation and mobility instructors involved in the multidisciplinary teams, as well as from the PA member.

Patients reported to have experienced the first signs of visual impairment during their early childhood. As also shown in other studies, ${ }^{15}$ parental caregivers were able to reliably recognize some of the earliest RPE65-related IRDs signs (light gazing, lack of eye contact, night blindness). Since confirming the clinical diagnosis may be challenging in early childhood, parental observation may be crucial for the clinical suspicion, and it may also help pediatricians and ophthalmologists to identify - or suspect - the condition and early refer patients to centers specialized in IRDs.

Patients reported that the communication of their clinical diagnosis was characterized by misleading messages, causing disorientation and sometimes the giving up of visual rehabilitation and follow-up visits; as suggested by the in-depth interviews, a psychological support from the communication of the diagnosis onward may help patients to proactively engage their care pathway. Communication issues can be further investigated and integrated with studies on the making-sense and coping with RPE65related IRDs. ${ }^{43,44}$ Conversely, clinicians from specialized centers reported a strong sense of identification with their patients or caregivers. They were also attentive to the nonclinical aspects of the communication: active listening, empathy, and the effort to give correct and realistic information that may debunk any misleading communication issues also reported by multidisciplinary healthcare professionals and the PA representative during the in-depth interviews, while engaging patients in a positive care relationship, as confirmed in the narratives and the parallel charts.

As for genetic testing, patients reported to have initially been not aware of its importance - due to young age or misleading or inadequate information after the clinical diagnosis. Genetic counselors emerged as critical to correctly support patients and caregivers in understanding the genetic diagnosis and the test result, and genetic counseling should be provided before and after testing; patients and caregivers should also be informed and prepared on the test limitations and the implications of the results. ${ }^{45}$ Conversely, in the narratives and the parallel charts, gene therapy undoubtedly emerged as a historic breakthrough, a chance to maintain or even improving the current visual ability, in line with other studies, ${ }^{46}$ which highlight its emotional impact on patients, caregivers, and even clinicians. However, as stressed in the parallel charts and as emerged in other studies, ${ }^{17}$ the eligibility criteria and the possibilities to access gene therapy should be further clarified, to ensure its applicability to as many patients as possible.

The narratives and the in-depth interviews confirmed that RPE65-related IRDs need a specialized approach for a successful evolution of the care pathway and an effective management of these conditions: specialized centers remain well distributed across Italy; thus, a structured network should also be implemented, to efficiently address patients' and caregivers' interregional mobility. Moreover, as suggested in other studies, ${ }^{17,18,47}$ a multidisciplinary approach is required to understand patients and provide them with support, also focusing on the psychosocial and organizational issues. In this perspective, the present project can provide preliminary suggestions to implement clinical practice:

(a) Building a robust and well-organized network, which also includes PAs, to support the patient's referral to IRDs specialized centers is crucial to facilitate the access to an early diagnosis and management, and to avoid challenging waiting times to initiate the rehabilitation and therapeutic pathway. Genetic counseling, provided by clinical geneticists specialized in IRDs, emerges as critical both before and after genetic testing, ${ }^{45}$ while gene therapy access and eligibility criteria should be further clarified. ${ }^{17}$ 
(b) A proper and empathetic communication is central: on the one hand, to effectively provide realistic information about the condition and its management, and, on the other, to engage patients so they could maintain an active perspective on the care pathway and to early access rehabilitation programs.

(c) The management of IRDs should be promoted by optimizing a multidisciplinary approach, which is essential to meet the complex care needs of each patient. ${ }^{18}$ The psychological support remains criti$\mathrm{cal}$, from the communication of the clinical diagnosis throughout the care pathway, and dedicated spaces may be provided to patients and their caregivers for them to share and exchange their illness experience. In addition, the role of the orientation and mobility instructor should be further acknowledged, for the purpose of a timely access to the rehabilitation path.

(d) The need for an increasing awareness of IRDs emerges as crucial on three levels: educational, for patients, caregivers, and relational networks; informative, for the general public; and professional, for pediatrics, ophthalmologists, and other professionals - from the first signs of the condition onward - to promote an early diagnosis and access to the care pathway.

The low annual incidence of RPE65-related IRDs approximately $1-2 \%$ of all IRD patients referred for genetic testing, with 4-5 new cases identified per year (unpublished data) - in Italy, ${ }^{13}$ where main centers specialized in IRDs and PA are jointly conducting a natural history study on these conditions, ${ }^{48}$ explains the low number of participating patients; nonetheless, the narratives collected suggest a strong dedication to the project, as well as a relationship of trust between patients, caregivers, and the healthcare professionals from the centers involved. Moreover, the data collection phase partially corresponded to the social distancing and local closings measures implemented by the Italian government to contain the Sars-Cov -2 pandemic, with consequences on the clinical follow-up and the participation in the project.

\section{Conclusions}

The project investigated RPE65-related IRDs care pathway as experienced by patients, caregivers, and healthcare professionals. Our results provide insights from the PA representatives and multidisciplinary healthcare professionals involved in this pathway. The integration and the analysis of these different perspectives through NM highlighted several preliminary areas of improvement in the management of RPE65-related IRDs, otherwise overlooked by the analysis of the clinical pathway only; finally, the nature of the project may stimulate further investigations on real-life experiences of the care pathway of these conditions.

\section{Abbreviations}

IRDs, inherited retinal disorders; RPE65, retinal pigment epithelium-specific $65 \mathrm{kDa}$ protein; RP, Retinitis Pigmentosa; LCA, Leber congenital amaurosis; WHO, World Health Organization; NM, Narrative Medicine; PA, Patient Association; ISTUD, Istituto Studi Direzionali; WCAG, Web Content Accessibility Guidelines; COREQ, Consolidated Criteria for Reporting Qualitative Research.

\section{Data Sharing Statement}

All datasets used and analyzed during the current research are available in Italian from the corresponding author, upon reasonable request.

\section{Ethics Approval}

The project was conducted according to the Declaration of Helsinki. The Ethical Committee of the Luigi Vanvitelli University Hospital (Naples, Italy) approved the project rationale, design, investigation questionnaires, and informed consent in September 2020.

\section{Consent to Participate}

Participants provided a web-based informed consent before their involvement and after being briefed on the purposes of the research and the procedures for the processing of personal data, according to General Data Protection Regulation of the European Union 2016/679 and the Italian Law 196/2003. The clinicians involved obtained a written informed consent to participate from the parents of underage patients during the first briefing on the project's methods and purposes. The informed consent included publication of anonymized responses and narratives.

\section{Acknowledgments}

The authors would also thank Giovanni Bosco Vitiello, Amelia Citro, Simona De Simone, Irene De Rienzo, 
Natalia Filimonova, Stefania Fortini, Cristiana Marchese, Maria Giulia Marini, Dario Pasquale Mucciolo, Vittoria Murro, Ilaria Passerini, and Simona Turco for their contributions to the deployment of the work. The authors wish to thank Novartis Farma, which sponsored and funded this work, in particular Vincenza Vinaccia for her editorial assistance. The authors would also like to thank Paolo Melillo from the Vanvitelli University Hospital for the support he provided in relation to the Ethical Committee's approval of the project, as well as the researchers of the Healthcare Area of the ISTUD Foundation for their useful role throughout the project, and all the people affected by an RPE65-related IRD, their caregivers and the healthcare professionals and Patient Association representatives who took part in the research. Acknowledgements are also extended to Enago (www.enago.com) for the English language review.

\section{Author Contributions}

All authors contributed to the project's conceptualization, investigation, and data analysis and validation. All authors contributed to data analysis, drafting or revising the article, gave final approval of the version to be published, agreed to the submitted journal, and agree to be accountable for all aspects of the work.

\section{Funding}

This work was funded by Novartis Farma.

\section{Disclosure}

FS received honoraria from Novartis Pharmaceuticals, Italy, for holding webinars and serving advisory boards. AS received honoraria from Novartis Pharmaceuticals, Italy, for serving on advisory boards. BF, received honoraria from Novartis Pharmaceuticals, Italy, for holding webinars and a lecture fee from UVET GBT, Milan, Italy. GB, and GI have received honoraria from Novartis Pharmaceuticals, Italy, for holding webinars; in addition GB received personal fees from Maple Health, personal fees from Kantar health, outside the submitted work. MA is employees of Novartis Pharmaceuticals, Italy, and Region Europe.

\section{References}

1. Ziccardi L, Cordeddu V, Gaddini L, et al. Gene therapy in retinal dystrophies. In $J$ Mol Sci. 2019;20(22):5722. doi:10.3390/ ijms20225722
2. Broadgate S, Yu J, Downes SM, Halford S. Unravelling the genetics of inherited retinal dystrophies: past, present and future. Prog Retin Eye Res. 2017;59:53-96. doi:10.1016/j.preteyeres.2017.03.003

3. Kang C, Scoo LJ. Voretigene neparvovec: a review in RPE65 mutation-associated inherited retinal dystrophy. Mol Diagn Ther. 2020;24(4):487-495. doi:10.1007/s40291-020-00475-6

4. Duncan JL, Pierce A, Laster AM, et al. Inherited retinal degenerations: current landscape and knowledge gaps. Transl Vis Sci Technol. 2018;7(4):6. doi:10.1167/tvst.7.4.6

5. Verbakel SK, van Huet RAC, Boon CJF, et al. Non-syndromic retinitis pigmentosa. Prog Retin Eye Res. 2018;66:157-186. doi:10.1016/j.preteyeres.2018.03.005

6. Kondkar AA, Abu-Amero KK. Leber congenital amaurosis: current genetic basis, scope for genetic testing and personalized medicine. Exp Eye Res. 2019;189:107834. doi:10.1016/j.exer.2019.107834

7. Prokofyeva E, Troeger E, Wilke R, Zrenner E. Early visual symptom patterns in inherited retinal dystrophies. Ophthalmologica. 2011;226 (3):151-156. doi:10.1159/000330381

8. Jacobson SG, Aleman T, Cideciyan AV, et al. Defining the residual vision in leber congenital amaurosis caused by RPE65 mutations. Invest Ophthalmol Vis Sci. 2009;50(5):2368-2375. doi:10.1167/ iovs.08-2696

9. Russell SR, Bennet J, Wellman JA, et al. Efficacy and safety of voretigene neparvovec (AAV2-hRPE65v2) in patients with RPE65-mediated inherited retinal dystrophy: a randomised, controlled, open-label, Phase 3 trial. Lancet. 2017;390(10097):849-860. doi:10.1016/S0140-6736(17)31868-8

10. Russell SR, Maguire AM, Bennett J, et al. Visual function questionnaire responses in the voretigene neparvovec phase 3 trial. Invest Ophthalmol Vis Sci. 2019;60(9):4968.

11. Hafler BP. Clinical progress in inherited retinal degenerations: gene therapy clinical trials and advances in genetic sequencing. Retina. 2017;37(3):417-423. doi:10.1097/IAE.0000000000001341

12. Thompson DA, Ali RR, Banin E, et al. Advancing therapeutic strategies for inherited retinal degeneration: recommendations from the monaciano symposium. Invest Ophthalmol Vis Sci. 2015;56 (2):918-931. doi:10.1167/iovs.14-16049

13. Lorenz B, Tavares J, van den Born LI, Marques JP, Scholl HPN; EVICR. net Group. Current management of patients with RPE65 mutation-associated inherited retinal degenerations (IRDs) in Europe. Results of a multinational survey by the European Vision Institute Clinical Research Network EVICR.net. Ophthalmic Res. 2021;64(5):740-753. doi:10.1159/000515688

14. Kurent A, Stirn-Kranjc B, Brecelj J. Electroretinographic characteristics in children with infantile nystagmus syndrome and early-onset retinal dystrophies. Eur $J$ Ophthalmol. 2015;25(1):33-42. doi:10.5301/ejo.5000493

15. Suppiej A, Marino S, Reffo ME, et al. Early onset retinal dystrophies: clinical clues to diagnosis for pediatricians. Ital J Pediatr. 2019;45 (1):168. doi:10.1186/s13052-019-0760-5

16. Mühlfriedel R, Sothilingam V, Tanimoto N, Seeliger MW. Gene replacement therapy for inherited retinal dystrophies. Klin Monbl Augenheilkd. 2017;234(3):320-328. doi:10.1055/s-0043-101817

17. Sodi A, Banfi S, Testa F, et al; Italian IRD Working Group. RPE65associated inherited retinal diseases: consensus recommendations for eligibility to gene therapy. Orphanet $J$ Rare Dis. 2021;16(1):257. doi:10.1186/s13023-021-01868-4

18. Parmeggiani F, Sato G, De Nadai K, Romano MR, Binotto A, Costagliola C. Clinical and rehabilitative management of retinitis pigmentosa: up-to-date. Curr Genomics. 2011;12(4):250-259. doi:10.2174/138920211795860125

19. Patel U, Boucher M, de Léséleuc L, Visintini S. Voretigene neparvovec: an emerging gene therapy for the treatment of inherited blindness. In: CADTH Issues in Emerging Health Technologies. Ottawa (ON): Canadian Agency for Drugs and Technologies in Health; Mar 1, 2018:2016-2169. 
20. Herse P. Retinitis pigmentosa: visual function and multidisciplinary management. Clin Exp Optom. 2005;88(5):335-350. doi:10.1111/ j.1444-0938.2005.tb06717.x

21. Gualandi R, Masella C, Viglione D, Tartaglini D, Frey R. Exploring the hospital patient journey: what does the patient experience? PLOS One. 2019;14(12):e0224899. doi:10.1371/journal.pone.0224899

22. Greenhalgh T. Cultural contexts of health: the use of narrative research in the health sector. Copenhagen: WHO Regional Office for Europe; 2016. Health Evidence Network (HEN) synthesis report 49. Available from: http://www.euro.who.int/_data/assets/pdf_file/0004/317623/ HEN-synthesis-report-49.pdf. Accessed March 23, 2021.

23. Pierret J. The illness experience: state of knowledge and perspectives for research. Sociol Health Illn. 2003;25:4-22. doi:10.1111/14679566.t01-1-00337

24. Ragusa L, Crinò A, Grugni G, Reale L, Fiorencis A, Licenziati MR. Caring and living with Prader-Willi syndrome in Italy: integrating children, adults and parents' experiences through a multicentre narrative medicine research. BMJ Open. 2020;10(8):e036502. doi:10.1136/bmjopen-2019-036502

25. Tonini MC, Fiorencis A, Iannacchero R, et al. Narrative medicine to integrate patients', caregivers' and clinicians' migraine experiences: the DRONE multicentre project. Neurol Sci. 2021;15:1-12.

26. Marini MG. Narrative Medicine: Bridging the Gap Between Evidence-Based Care and Medical Humanities. London: Springer International Publishing; 2016.

27. Kleinman A. The Illness Narrative, Suffering and Healing the Human Condition. New York: Basic Book; 1989.

28. Greenhalgh T, Hurwitz B. Narrative based medicine: why study narrative? $\quad B M J . \quad 1999 ; 318(7175): 48-50 . \quad$ doi:10.1136/ bmj.318.7175.48

29. Midena E, Varano M, Pilotto E, et al. Real-life patient journey in neovascular age-related macular degeneration: a narrative medicine analysis in the Italian setting. Eye. 2021. doi:10.1038/s41433-021-01470-9

30. Fioretti C, Mazzocco K, Riva S, Oliveri S, Masiero M, Pravettoni G. Research studies on patients' illness experience using the narrative medicine approach: a systematic review. BMJ Open. 2016;6(7): e011220. doi:10.1136/bmjopen-2016-011220

31. Marini MG. Languages of Care in Narrative Medicine. Words, Space and Time in the Healthcare Ecosystem. London: Springer International Publishing; 2019.

32. Nowaczyk MJ. Narrative medicine in clinical genetics practice. $A m$ $J$ Med Genet A. 2012;158A(8):1941-1947. doi:10.1002/ajmg.a.35482

33. Web Content Accessibility Guidelines (WCAG) 2.1. W3C Recommendations; June 05, 2018. Available from: https://www.w3. org/TR/WCAG21/. Accessed March 23, 2021.

34. Reid K, Soundy A. A qualitative study examining the illness narrative master plots of people with head and neck cancer. Behav Sci. 2019;9(10):110. doi:10.3390/bs9100110

35. Peeters B, Marini M. Narrative medicine across languages and cultures: using minimal English for increased comparability of patients' narratives. In: Goddard C, editor. Minimal English for a Global World: Improved Communication Using Fewer Words. Basingstoke, UK: Palgrave Macmillan; 2018:259-286.
36. Charon R. The patient-physician relationship. Narrative Medicine: a model for empathy, reflection, profession, and trust. JAMA. 2001;286(15):1897-1902. doi:10.1001/jama.286.15.1897

37. Banfi P, Cappuccio A, Latella ME, et al. Narrative medicine to improve the management and quality of life of patients with COPD: the first experience applying parallel chart in Italy. Int J Chron Obstruct Pulmon Dis. 2018;13:287-297. doi:10.2147/ COPD.S148685

38. Brédart A, Marrel A, Abetz-Webb L, Lasch K, Acquadro C. Interviewing to develop Patient-Reported Outcome (PRO) measures for clinical research: eliciting patients' experience. Health Qual Life Outcomes. 2014;5(12):15. doi:10.1186/1477-7525-12-15

39. Regulation (EU) 2016/679 of the European Parliament and of the Council of 27 April 2016 on the protection of natural persons with regard to the processing of personal data and on the free movement of such data and repealing Directive 95/46/EC (General Data Protection Regulation. Published on the Official Journal of the European Union L 119; May 4, 2016. Available from: https://eur-lex.europa.eu/legalcontent/EN/TXT/PDF/?uri=CELEX:32016R0679. Accessed March 23, 2021

40. Personal data code protection. Legislat. Decree no. 196 of 30 June 2003. Published on the Italian Official Journal n. 174. Supplementary n. 123; July 29, 2003. Available from: https://www. camera.it/parlam/leggi/deleghe/Testi/03196dl.htm. Accessed March 23, 2021.

41. Bazeley P, Jackson K. Qualitative Data Analysis with NVivo. London: SAGE; 2013.

42. Tong A, Sainsbury P, Craig J. Consolidated criteria for reporting qualitative research (COREQ): a 32-item checklist for interviews and focus groups. Int J Qual Health Care. 2007;19:6. doi:10.1093/ intqhe/mzm042

43. Thurston M, Thurston A, McLeod J. Socio-emotional effects of the transition from sight to blindness. $B r \quad J$ Vis Impair. 2010;28 (2):90-112. doi:10.1177/0264619609359304

44. Bittner AK, Edwards L, George M. Coping strategies to manage stress related to vision loss and fluctuations in retinitis pigmentosa. Optometry. 2010;81(9):461-468. doi:10.1016/j.optm.2010.03.006

45. American Academy of Ophthalmology. Clinical statement: recommendations on clinical assessment of patients with inherited retinal degenerations; 2016. Available from: https://www.aao.org. Accessed May 10, 2021.

46. Daiger SP, Bowne SJ, Sullivan LS. Perspective on genes and mutations causing retinitis pigmentosa. Arch Ophthalmol. 2007;125 (2):151-158. doi:10.1001/archopht.125.2.151

47. Garip G, Kamal A. Systematic review and meta-synthesis of coping with retinitis pigmentosa: implications for improving quality of life. BMC Ophthalmol. 2019;19(1):181. doi:10.1186/s12886-019-1169-z

48. University of Campania "Luigi Vanvitelli", Natural History of Patients With Inherited Retinal Diseases Due to Mutations in RPE65 Gene (RPE65-NHS), Identifier NCT04525261, https://clinical trials.gov/ct2/show/NCT04525261, last accessed on November 25, 2021
Clinical Ophthalmology

\section{Publish your work in this journal}

Clinical Ophthalmology is an international, peer-reviewed journal covering all subspecialties within ophthalmology. Key topics include: Optometry; Visual science; Pharmacology and drug therapy in eye diseases; Basic Sciences; Primary and Secondary eye care; Patient Safety and Quality of Care Improvements. This journal is indexed on PubMed
Central and CAS, and is the official journal of The Society of Clinical Ophthalmology (SCO). The manuscript management system is completely online and includes a very quick and fair peer-review system, which is all easy to use. Visit http://www.dovepress.com/ testimonials.php to read real quotes from published authors. 